\title{
SEMANTIC SEGMENTATION OF CONVOLUTIONAL NEURAL NETWORK FOR SUPERVISED CLASSIFICATION OF MULTISPECTRAL REMOTE SENSING
}

\author{
Xue Lei $^{1}$, Liu Chang ${ }^{1}$, Wu Yunqiang ${ }^{1}$, Li Huiying ${ }^{1,2^{*}}$ \\ ${ }^{1}$ Computer science and technology department, Jilin University, 130012 ChangChun, Jilin province, China \\ ${ }^{2}$ Symbol Computation and Knowledge Engineer of Ministry of Education, Jilin University, 130012 Changchun, Jilin province, \\ China
}

KEY WORDS: Semantic Segmentation, Multi Spectral Remote Sensing, Convolutional Neural Network, U-net, multi-scale image

\begin{abstract}
:
Semantic segmentation is a fundamental research in remote sensing image processing. Because of the complex maritime environment, the classification of roads, vegetation, buildings and water from remote Sensing Imagery is a challenging task. Although the neural network has achieved excellent performance in semantic segmentation in the last years, there are a few of works using CNN for ground object segmentation and the results could be further improved. This paper used convolution neural network named U-Net, its structure has a contracting path and an expansive path to get high resolution output. In the network, We added BN layers, which is more conducive to the reverse pass. Moreover, after upsampling convolution, we add dropout layers to prevent overfitting. They are promoted to get more precise segmentation results. To verify this network architecture, we used a Kaggle dataset. Experimental results show that U-Net achieved good performance compared with other architectures, especially in high-resolution remote sensing imagery.
\end{abstract}

\section{INTRODUCTION}

In recent years, some classifiers have a good performance in the image classification, such as minimum-distance classifier, Support Vector Machine (SVM), PCA linear dimension reduction method and Mean clustering method. However, their success was limited in feature extraction and classification of multispectral images.

In the last two years, deep convolutional networks have outperformed the state of the art in many visual recognition tasks. According to the characteristics of multispectral images, the convolutional network model with multi-layer perceptrons can be designed to solve this classification problem by using the spectral information and spatial information of the data.

In this paper, we present a classification method of multispectral images with improved U-net network. U-Net is an encoder-decoder structure in which the encoder gradually reduces the spatial dimension of the pooling layer and the decoder gradually fixes the details and spatial dimensions of the object. There is usually a quick connection between the encoder and the decoder, so it helps the decoder to better fix the details of the target, especially in high-resolution remote sensing imagery. We classify 10 kinds of ground objects appearing in remote sensing images, and use some methods of graphics to post-process the preliminary classification results to make the classification results more accurate.

\section{METHOD}

\subsection{Improved Network Architecture}

By constructing a U-net based convolutional network model, the end-to-end correspondence of low-resolution features can be effectively achieved. U-net network combines layers of the feature hierarchy and refines the spatial precision of the output.

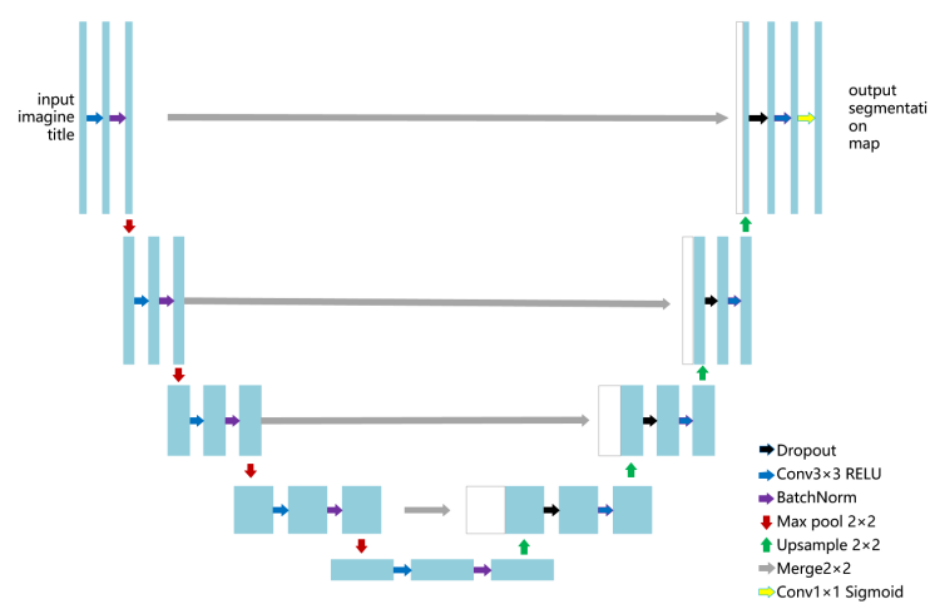

Figure 1. Our U-net architecture

\footnotetext{
* Correspoding author Huiying Li, Email: lihuiying@ jlu.edu.cn
} 
The U-net network structure consists of a contracting path and an expansive path. The contracting path follows the typical architecture of a convolutional network. In total the network has 23 convolutional layers. It consists of the repeated application of two $3 \times 3$ convolutions (unpadded convolutions), each followed by a rectified linear unit (ReLU) and a $2 \times 2$ max pooling operation with stride 2 for downsampling. After each convoluted layer, we add a BN layer. Every step in the expansive path consists of an upsampling of the feature map followed by a $2 \times 2$ convolution (up-conv) that halves the number of feature channels, a concatenation with the correspondingly cropped feature map from the contracting path, and two $3 \times 3$ convolutions, each followed by a ReLU. After upsamping convolution, we add a dropout layer to prevent overfitting. At the final layer a $1 \times 1$ convolution is used to map each components feature vector to the desired number of classes (shown in Figure 1).

\subsection{Data preprocessing}

We have marked 10 basic objects, including housing, track, tree, etc. (Figure 2 and Table1)

a. Firstly, we will transform the coordinate, geographical coordinates of corresponding to the pixel coordinates of the picture one by one. The coordinate information is connected into an irregular polygon area, and the interior of the polygon is filled to generate a binary mask image. Housing and road renderings are as follows (Figure 3). b. And then we combine the multi-scale image block with the sliding window generation method. The block overlap technique is used to cover the edge of the whole image. The original image and its corresponding binary mask are used as the input of the network.

c. For seven kinds of ground objects, such as houses, artificial buildings, railways, trees, crops, roads and waterlogged areas, the training set is a multi-spectral band image with 16 channels, and the size of the image is set as 8. With the goal of minimizing the sum of binary cross-entropy losses, train each category accordingly, simply average the output of all models, and then post-process the parameters according to a particular category.

d. For rivers and watersheds, a combination of linear regression and random forest can be used to identify the river and train the 8-channel input data, which works well due to the unique spectrum of water. Later post-processing by combining the indifferent moisture index and the apical chlorophyll content index gives accurate results.

e. For large and small vehicles, due to the large resolution of the training images, the amount of data relative to the vehicle is very small, we must use special means for training. Using the corresponding RGB band image data, averaging, training a fusion network, the vehicle separate segmentation.
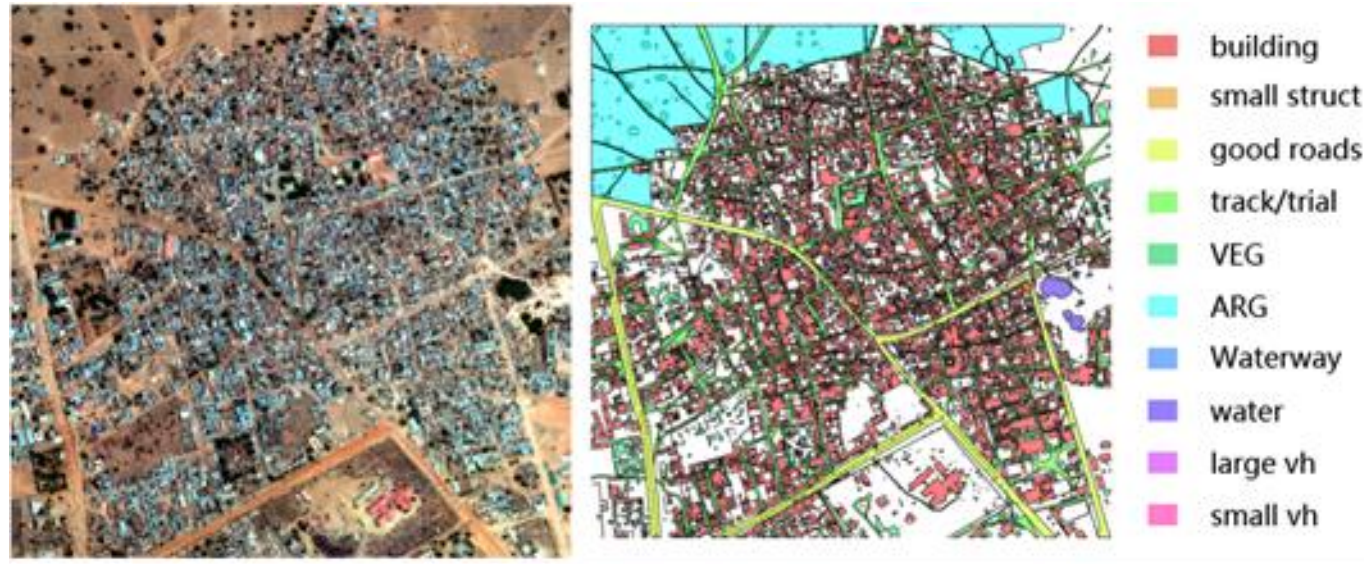

Fig.2 Segmentation result with manual ground truth
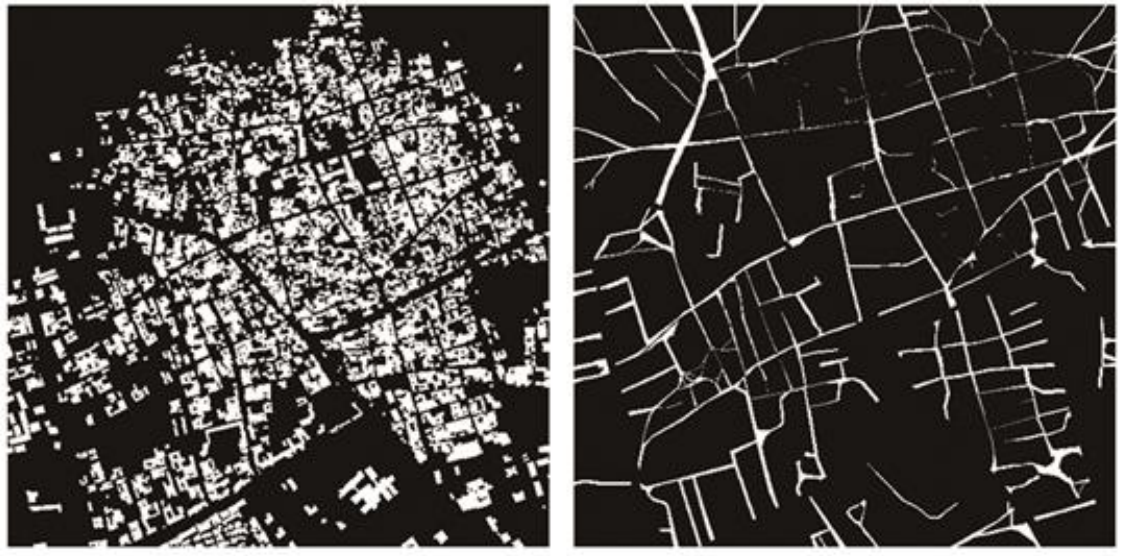

Fig.3. Preprocessing of input data. 
The International Archives of the Photogrammetry, Remote Sensing and Spatial Information Sciences, Volume XLII-3, 2018 ISPRS TC III Mid-term Symposium “Developments, Technologies and Applications in Remote Sensing”, 7-10 May, Beijing, China

\begin{tabular}{|c|c|c|c|c|}
\hline Type & Band & Pixel resolution & Channels & Size \\
\hline 3 channels & RGB & $0.31 \mathrm{~m}$ & 3 & $3348 \times 3392$ \\
\hline grayscale & Panchromatic Band & $0.31 \mathrm{~m}$ & 1 & $3348 \times 3392$ \\
\hline 16 channels & Multispectral & $1.24 \mathrm{~m}$ & 8 & $837 \times 848$ \\
\hline 16 channels & Near-infrared band & $7.5 \mathrm{~m}$ & 8 & $134 \times 136$ \\
\hline
\end{tabular}

Table 1. Parameters of image.

\section{EXPERIMENTAL RESULTS}

As shown in figure 4, a preliminary identification result of all categories of an image:

The first line displays the second to sixth channel images of original image. Line 2 and line 3 are the recognitions of each category, with red dots for the area identified, among the third figure of the second row and the Fig 3, 4, 5 of the third row show no results to identify the target figure:

As shown in Fig.5, the picture on the left is original, and the picture on the right shows the final result of the initial recognition result after the post-processing, in which the target marked by white area.

The result of house type identification: by contrasting the effect of the left and right pictures, it can be clearly seen that each block is identified in the right picture, and even the object partially shielded by trees is effectively identified(shown in Figure 5.a).
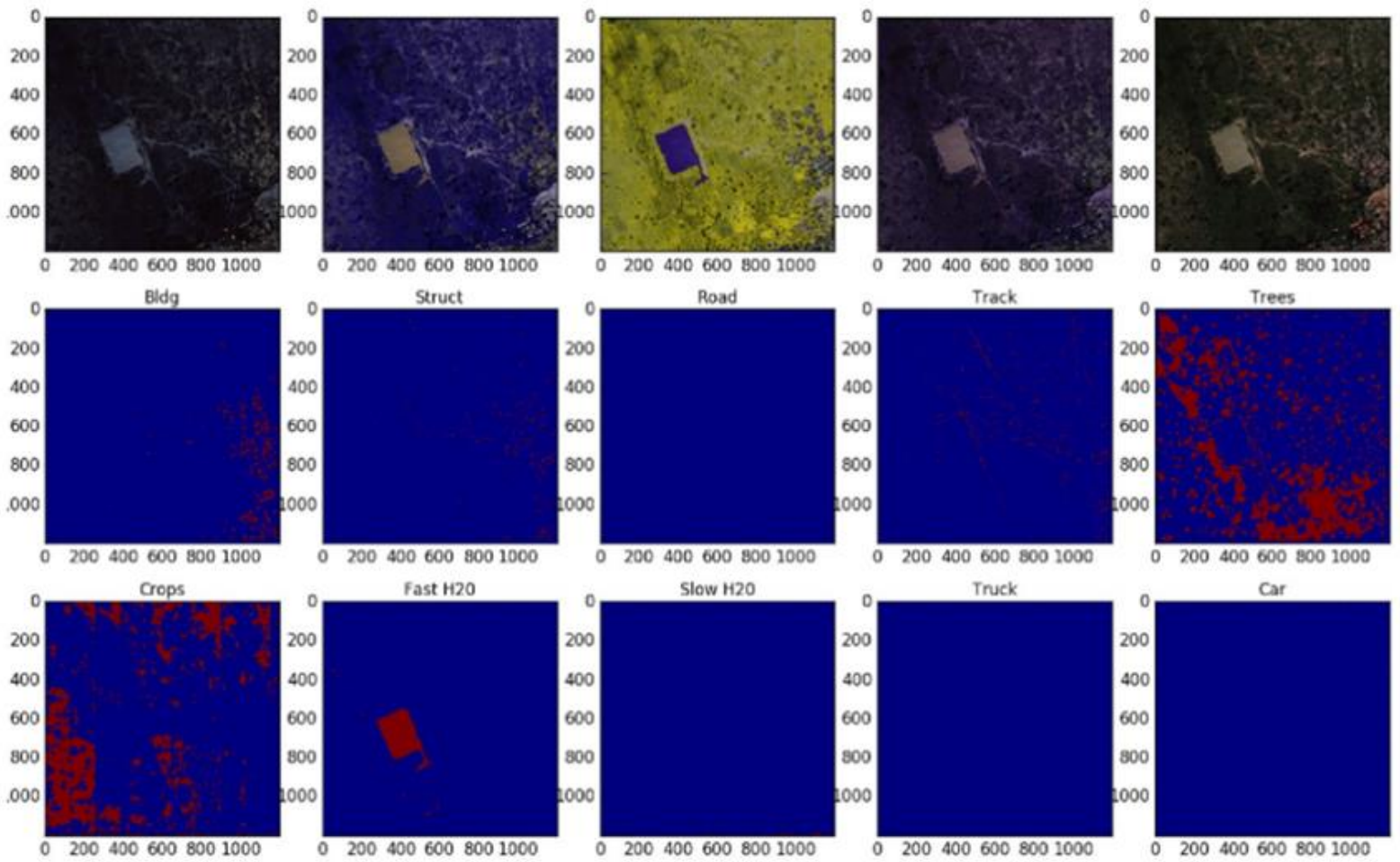

Fig.4. Initial preliminary identification of all categories of images. 


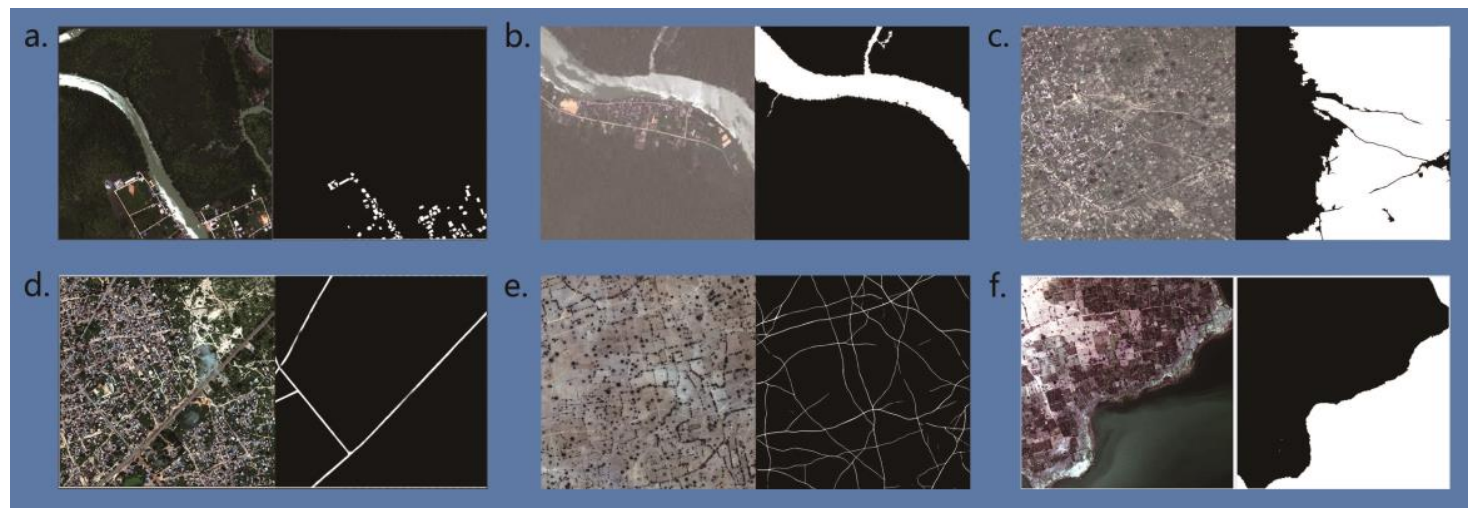

Fig.5. The results of identification

\section{EVALUATION OF RESULTS}

The data used in this experiment is a set of multi-spectral remote sensing images provided by the German laboratory DSTL on Kaggle. To verify the accuracy of the experimental results, first calculate the Jacad index of all classes of training data, and then use the network model to evaluate Training data, and then submit the assessment results to kaggle backstage for verification. Table 2 shows the corresponding Jacad coefficient for each type of feature in the training data.

\begin{tabular}{|c|c|c|c|}
\hline Category & Training area & $\begin{array}{c}\text { Polygonal } \\
\text { area }\end{array}$ & $\begin{array}{c}\text { Jaccard } \\
\text { coefficient }\end{array}$ \\
\hline Housing & $6.843670 \mathrm{e}-05$ & 0.002070227 & $3.305758 \mathrm{e}-02$ \\
\hline $\begin{array}{c}\text { Small } \\
\text { building }\end{array}$ & $1.460820 \mathrm{e}-05$ & 0.002070227 & $7.056327 \mathrm{e}-03$ \\
\hline Main road & $1.674853 \mathrm{e}-05$ & 0.002070227 & $8.090188 \mathrm{e}-03$ \\
\hline Other trails & $6.217386 \mathrm{e}-05$ & 0.002070227 & $3.003239 \mathrm{e}-02$ \\
\hline Trees & $2.106242 \mathrm{e}-04$ & 0.002070227 & $1.017396 \mathrm{e}-01$ \\
\hline Crops & $5.706590 \mathrm{e}-04$ & 0.002070227 & $2.756504 \mathrm{e}-01$ \\
\hline River & $1.023189 \mathrm{e}-05$ & 0.002070227 & $4.942400 \mathrm{e}-03$ \\
\hline Water area & $3.488082 \mathrm{e}-06$ & 0.002070227 & $1.684879 \mathrm{e}-03$ \\
\hline Small cars & $7.719743 \mathrm{e}-08$ & 0.002070227 & $3.728935 \mathrm{e}-05$ \\
\hline Large cars & $3.160106 \mathrm{e}-07$ & 0.002070227 & $1.526454 \mathrm{e}-04$ \\
\hline
\end{tabular}

Table 2 The Jaccard coefficient of training data

In the above table, we can see that the Category 5 trees accounted for $0.1 \%$ of the total, the Category 6 crops accounted for 0.27 as a whole, and the other categories accounted for very few ratios. For these classification results, the size of the Jaccard coefficient is equal to the category. The size of the image area occupied, so we only need to obtain the Jaccard coefficient of each category, we can determine the experimental recognition effect. Table 3 shows the results of the experiment given by the kaggle background.

\begin{tabular}{|c|c|c|c|}
\hline No. & Category & Public Score & Private Score \\
\hline 1 & Housing & 0.07732 & 0.06102 \\
\hline 2 & Small building & 0.01832 & 0.02126 \\
\hline 3 & Main road & 0.07693 & 0.05522 \\
\hline 4 & Other trails & 0.03765 & 0.04114 \\
\hline 5 & Trees & 0.05102 & 0.06371 \\
\hline 6 & Crops & 0.08079 & 0.08137 \\
\hline 7 & River & 0.09645 & 0.08972 \\
\hline 8 & Water area & 0.06179 & 0.04598 \\
\hline 9 & Small cars & 0.03154 & 0.00747 \\
\hline 10 & Large cars & 0.00311 & 0.00429 \\
\hline Final recognition accuracy & 0.53129 & 0.46937 \\
\hline
\end{tabular}

Table 3 Experimental identification results
The public score is the result of verification using $19 \%$ of the test data, and the private score is the verification result of the remaining $81 \%$ of the data. It is not difficult to find that the recognition rates for houses, road trunk lines, crops and rivers are relatively high, and the recognition rates for automobiles, artificial irregular buildings and artificial trails are relatively low, especially for the identification of large-scale automobiles.

\section{ACKNOWLEDGEMENTS}

This study was supported by Scientific and Technological Development Scheme of Jilin Province (Grant No. 20180101048JC), Major Special Project of China Highresolution Earth Observation System (CHEOS) (04-Y20A359001-15/17)

\section{REFERENCES}

Castelluccio, Marco, et al. "Land use classification in remote sensing images by convolutional neural networks." arXiv preprint arXiv:1508.00092 (2015).

Chen, Yushi, Xing Zhao, and Xiuping Jia. "Spectral-spatial classification of hyperspectral data based on deep belief network." IEEE Journal of Selected Topics in Applied Earth Observations and Remote Sensing 8.6 (2015): 2381-2392.

Cheng, Gong, Peicheng Zhou, and Junwei Han. "Learning rotation-invariant convolutional neural networks for object detection in VHR optical remote sensing images." IEEE Transactions on Geoscience and Remote Sensing 54.12 (2016): 7405-7415.

Heermann, Philip Dale, and Nahid Khazenie. "Classification of multispectral remote sensing data using a back-propagation neural network." IEEE Transactions on Geoscience and Remote Sensing 30.1 (1992): 81-88.

$\mathrm{Hu}$, Wei, et al. "Deep convolutional neural networks for hyperspectral image classification." Journal of Sensors 2015 (2015).

Kussul, Nataliia, et al. "Deep learning classification of land cover and crop types using remote sensing data." IEEE Geoscience and Remote Sensing Letters 14.5 (2017): 778-782.

Makantasis, Konstantinos, et al. "Deep supervised learning for hyperspectral data classification through convolutional neural networks." Geoscience and Remote Sensing Symposium (IGARSS), 2015 IEEE International. IEEE, 2015. 
The International Archives of the Photogrammetry, Remote Sensing and Spatial Information Sciences, Volume XLII-3, 2018 ISPRS TC III Mid-term Symposium "Developments, Technologies and Applications in Remote Sensing", 7-10 May, Beijing, China

Vakalopoulou, Maria, et al. "Building detection in very high resolution multispectral data with deep learning features." Geoscience and Remote Sensing Symposium (IGARSS), 2015 IEEE International. IEEE, 2015.

Wang, Lizhe, et al. "Spectral-spatial multi-feature-based deep learning for hyperspectral remote sensing image classification." Soft Computing 21.1 (2017): 213-221.

Zhang, Liangpei, Lefei Zhang, and Bo Du. "Deep learning for remote sensing data: A technical tutorial on the state of the art." IEEE Geoscience and Remote Sensing Magazine 4.2 (2016): $22-40$.

Zou, Qin, et al. "Deep learning based feature selection for remote sensing scene classification." IEEE Geoscience and Remote Sensing Letters 12.11 (2015): 2321-2325.

Zhang, Liangpei, et al. "Deep learning for remote sensing image understanding." Journal of Sensors 2016 (2016).

Zhao, Wenzhi, et al. "On combining multiscale deep learning features for the classification of hyperspectral remote sensing imagery." International Journal of Remote Sensing 36.13 (2015): 3368-3379. 